\title{
A rare $C H D 5$ haplotype and its interactions with environmental factors predicting hepatocellular carcinoma risk
}

\author{
Qin Xiao ${ }^{1,2+}$, Lianzhou Chen ${ }^{3 \dagger}$, Haiqing Luo ${ }^{1,4 \dagger}$, Hongmei Li ${ }^{1,5 \dagger}$, Qingming Kong ${ }^{6}$, Fei Jiao ${ }^{7}$, Shifeng Pang ${ }^{1}$, \\ Ming Zhang ${ }^{8}$, Feifei Lan $^{9}$, Wenguo Fan ${ }^{10}$, Hui Luo ${ }^{1^{*}}$, Tao Tao ${ }^{8^{*}}$ and Xiao Zhu ${ }^{1 *}$
}

\begin{abstract}
Background: CHD5 is a conventional tumour-suppressing gene in many tumours. The aim of this study was to determine whether CHD5 variants contribute to the risk of hepatocellular carcinoma (HCC).

Methods: Gene variants were identified using next-generation sequencing targeted on referenced mutations followed by TaqMan genotyping in two case-control studies.

Results: We discovered a rare variant (haplotype AG) in CHD5 (rs12564469-rs9434711) that was markedly associated with the risk of HCC in a Chinese population. A logistical regression model and permutation test confirmed the association. Indeed, the association quality increased in a gene dose-dependent manner as the number of samples increased. In the stratified analysis, this haplotype risk effect was statistically significant in a subgroup of alcohol drinkers. The false-positive report probability and multifactor dimensionality reduction further supported the finding.

Conclusions: Our results suggest that the rare CHD5 gene haplotype and alcohol intake contribute to the risk of HCC. Our findings can be valuable to researchers of cancer precision medicine looking to improve diagnosis and treatment of HCC.
\end{abstract}

Keywords: CHD5, Gene haplotype, Hepatocellular carcinoma, Alcohol intake, Risk

\section{Background}

Hepatocellular carcinoma (HCC) is the most common primary liver cancer and has the worst prognoses of all malignancies. The etiological background of HCC patients differs between patients from different regions. In China, chronic hepatitis B virus (HBV) infection is the most important risk factor for $\mathrm{HCC}$; two-thirds of the worldwide HBV carriers are Chinese, and approximately $20 \%$ of them have a chronic HBV infection [1].

Chromodomain helicase DNA-binding protein 5 (CHD5) is on the Homo sapiens chromosome 1p36.31. It

\footnotetext{
* Correspondence: luohui@gdmu.edu.cn; tao_tao79@163.com; bioxzhu@yahoo.com

${ }^{\dagger}$ Qin Xiao, Lianzhou Chen, Haiqing Luo and Hongmei Li contributed equally to this work.

${ }^{1}$ Guangdong Provincial Key Laboratory of Medical Molecular Diagnostics, Dongguan Scientific Research Center, Guangdong Medical University, Dongguan, China

${ }^{8}$ Department of Gastroenterology, Zibo Central Hospital, Zibo, China Full list of author information is available at the end of the article
}

is one of the nine members of the CHD-binding enzymes and belongs to the snf2 DNA helicase/methylase superfamily [2]. CHD5 consists of 42 exons coding for a $223 \mathrm{kDa}$ protein. Based on its protein sequence, it contains two PHD zinc fingers, two chromodomains and a helicase/ATPase domain.

Evidence that CHD5 functions as a tumour suppressor in human cancers has emerged principally from studies of neuroblastoma, wherein loss of the CHD5 locus on chromosome $1 \mathrm{p} 36.3$ is very common. CHD5 has garnered considerable interest owing to its ability to severely impact clonogenicity and tumourigenecity. Although its expression was thought to be restricted to neural-related tissues, it was subsequently found to be a tumour suppressor in neuroblastoma [3], melanoma [4], lung cancer [5], breast cancer [6], ovarian cancer [7], gastric cancer [8], colorectal cancer [9] and HCC [10]. CHD5 loss leads to a wide range of cellular consequences, and it, therefore, remains a promising

(c) The Author(s). 2018 Open Access This article is distributed under the terms of the Creative Commons Attribution 4.0 International License (http://creativecommons.org/licenses/by/4.0/), which permits unrestricted use, distribution, and reproduction in any medium, provided you give appropriate credit to the original author(s) and the source, provide a link to the Creative Commons license, and indicate if changes were made. The Creative Commons Public Domain Dedication waiver (http://creativecommons.org/publicdomain/zero/1.0/) applies to the data made available in this article, unless otherwise stated. 
candidate for further investigation in HCC. In this study, we tested the hypothesis that single-nucleotide polymorphisms (SNPs) in the 1 p36 region of CHD5 are associated with HCC.

\section{Methods}

\section{Study subjects}

First, 280 unrelated HCC patients and 255 healthy controls (admitted to the Zibo Central Hospital in North China between 2006 and 2010) were recruited for our study. Then, 549 HCC patients and 510 controls (admitted to the Peking University Shenzhen Hospital between 2007 and 2010, the First Affiliated Hospital at the Sun Yat-Sen University between 2007 and 2015, and the Cancer Hospital of Guangzhou Medical University between 2009 and 2011 in South China) were enrolled in the replication study. The selection criteria for the controls included no individual/family history of cancer or diabetes; no history of HBV, HCV, tuberculosis or HIV infection and frequency of age ( \pm 5 years) and sex matching those of the patients. All patients were newly diagnosed, previously untreated (no radiotherapy or chemotherapy) and were proven to have no other tumours. We used published diagnostic criteria for HCC [11, 12]. The definition of 'Ever or current smokers' is those who had smoked more than 100 cigarettes, which is equal to five packs in their whole life before the date they were diagnosed with cancer or before the date they were interviewed for the controls [13, 14]. The definition of 'Ever or current drinkers' were those who have consumed alcoholic beverages $\geq$ one time per week for 6 months or more previously; otherwise, they were defined as non-drinkers [15]. The purpose of frequency matching was to control confounding factors while evaluating the main effect of $\mathrm{CHD} 5$ polymorphisms. All patients and controls were Han Chinese in origin and lived in China. Relevant biographical features of the subjects are summarised in Table 1.

The committee of ethics in Guangdong Medical University authorised the experimental and research protocols of this study. Experiments on humans were performed in accordance with relevant guidelines and regulations. After clearly explaining the purpose of the study to the participants, all controls and patients (or relatives of patients who already died) provided written informed consent. The study also adhered to tenets in the Helsinki declaration. All potential participants who declined to participate or ended up not participating were eligible for treatment, and non-participation did not result in any disadvantages for patients.

\section{Targeted next-generation sequencing (NGS) and identification of genetic variants}

Aliquots of buffy coat and plasma separated from blood samples were stored at $-80{ }^{\circ} \mathrm{C}$ until subsequent treatment. All samples were included in the combined study. Genomic DNA was extracted from peripheral whole blood cells using the QIAamp system (QIAGEN Co.). Genomic DNA from 255 controls and $280 \mathrm{HCC}$ patients were randomly sheared by sonication to an average size of $250 \mathrm{bp}$ per fragment. Target enrichment technology was used as described by Anna Kiialainen et al. [16] The enriched libraries were loaded onto the HiSeq system 2000 and approximately 90 -bp paired-end reads were produced using the NGS technology (Illumina Genome Analyzer). We will use fastq short reads to align the NCBI build 37.1 hg19 [17]. Single-nucleotide variants (SNV) that obey the criteria that a. $P$ for Hardy-Weinberg equilibrium (HWE, $<10^{-4}$ ), b. duplicated paired-end reads, c. overall depth $\leq 8 \times$, d. SNP within 10 bp of a gap, or e. copy number variant $\geq 2$ were then filtered [18]. For these concerns, only qualified SNPs were considered for this evaluation, so a 164-SNP set was used for the primary case-control study. Plink was used to calculate single-nucleotide variants [19], and the Haploview was used to perform visualisation [20].

\section{Population risk evaluation, linkage disequilibrium (LD) mapping and gene-gene interactions}

We used the chi-square and Mann-Whitney $U$ tests to compare and evaluate the clinical data between the patients and controls in discovery, replication and the combined groups. The risk evaluation was assessed using the Pearson chi-square test. Because 164 SNPs were genotyped, the Bonferroni-corrected $P$ value for association studies is $0.05 / 164=0.0003$ for single SNPs.

A gene-gene interaction in this study is defined as an SNP-SNP interaction and was conducted with LD mapping. To estimate the degree of LD between pairs of loci, the standardised disequilibrium coefficient $\left(D^{\prime}\right)$ was calculated and haplotype blocks were defined using the Haploview programme [20]. The haplotypic imputation, reconstruction and frequency estimations were conducted with an expectation-maximisation algorithm [21]. $\mathrm{n}_{\mathrm{e}}=1 / \sum_{P \mathrm{i}}{ }^{2}$ was used to calculate the number of effective haplotypes, and Pi was the estimate of individual haplotype frequency [22]. Pi was calculated because the phase of the genotype was known and it was chosen in compliance with the homologous probabilities of occurrence that had a higher likelihood ( $>0.95$ as cut-point).

\section{Permutation test and quantile-quantile (Q-Q) analysis}

We performed permutation tests for $10^{5}$ permutations, in which subjects' phenotypes were randomly realigned. $P$ values (permutation or empirical $P$ values) were specified as permutation values that were at least as extreme as the original statistics divided by the total permutation numbers. For better estimation of empirical $P$ values, SNPs were reconsidered with $10^{5}$ permutations. Permutations 
Table 1 Clinical and laboratory features of the subjects included in the study

\begin{tabular}{|c|c|c|c|c|c|c|c|c|c|}
\hline \multirow[t]{2}{*}{ Characteristics } & \multicolumn{3}{|c|}{ Discovery study } & \multicolumn{3}{|c|}{ Replication study } & \multicolumn{3}{|c|}{ Combined study } \\
\hline & Cases (\%) & Controls (\%) & $P$ & Cases (\%) & Controls (\%) & $P$ & Cases (\%) & Controls (\%) & $P$ \\
\hline$n$ & 280 & 255 & & 549 & 510 & & 829 & 765 & \\
\hline Age $(y s$, mean $\pm S D)$ & $55.1 \pm 14.6$ & $41.5 \pm 9.1$ & $<0.001^{\mathrm{a}}$ & $56.6 \pm 11.3$ & $47.2 \pm 10.7$ & $<0.001^{\mathrm{a}}$ & $56.0 \pm 13.6$ & $44.8 \pm 10.3$ & $<0.001^{\mathrm{a}}$ \\
\hline Gender (F/M) & $53 / 227$ & $91 / 164$ & $<0.001^{b}$ & $125 / 424$ & $167 / 343$ & $<0.001^{b}$ & $178 / 651$ & $258 / 507$ & $<0.001^{b}$ \\
\hline Smoking & $99(35.36)$ & $56(21.96)$ & $0.001 b$ & $231(42.08)$ & $145(28.43)$ & $<0.001^{\mathrm{b}}$ & $330(39.81)$ & $201(26.27)$ & $<0.001^{\mathrm{b}}$ \\
\hline Missing & $5(1.79)$ & $7(2.75)$ & & $22(4.01)$ & $26(5.10)$ & & $27(3.26)$ & $33(3.98)$ & \\
\hline Drinking & $95(33.93)$ & $54(21.18)$ & $0.001^{b}$ & $210(38.25)$ & $129(25.29)$ & $<0.001^{\mathrm{b}}$ & 305 (36.79) & $183(23.92)$ & $<0.001 b$ \\
\hline Missing & $8(2.86)$ & $7(2.75)$ & & $28(5.10)$ & $29(5.69)$ & & $36(4.34)$ & $36(4.71)$ & \\
\hline $\mathrm{HBsAg}+$ & $224(80.00)$ & $0(0.00)$ & & 419 (76.32) & $0(0.00)$ & & $643(77.56)$ & & \\
\hline Anti-HCV & $0(0.00)$ & $0(0.00)$ & & $4(0.73)$ & $0(0.00)$ & & $4(0.48)$ & $0(0.00)$ & \\
\hline Anti-HIV & $0(0.00)$ & $0(0.00)$ & & $2(0.36)$ & $0(0.00)$ & & $2(0.24)$ & $0(0.00)$ & \\
\hline Serum AFP (>25 $\mu \mathrm{g} / \mathrm{L})$ & $233(83.21)$ & $0(0.00)$ & & $431(78.51)$ & $0(0.00)$ & & $664(80.10)$ & $0(0.00)$ & \\
\hline \multicolumn{10}{|l|}{ Tumor size (cm) } \\
\hline$\leq 5$ & $65(23.21)$ & & & $139(25.32)$ & & & $204(24.61)$ & & \\
\hline$>5, \leq 10$ & $93(33.21)$ & & & $273(49.73)$ & & & $366(44.15)$ & & \\
\hline$>10$ & $122(43.57)$ & & & $137(24.95)$ & & & $259(31.24)$ & & \\
\hline \multicolumn{10}{|l|}{ Cirrhosis } \\
\hline No & $16(5.71)$ & & & $38(6.92)$ & & & $54(6.51)$ & & \\
\hline Yes & 260 (92.86) & & & $504(91.80)$ & & & $764(92.16)$ & & \\
\hline Missing & $4(1.43)$ & & & $7(1.28)$ & & & $11(1.33)$ & & \\
\hline \multicolumn{10}{|l|}{ Tumor morphology } \\
\hline No residual tumor & 19 (6.79) & & & $43(7.83)$ & & & $62(7.48)$ & & \\
\hline Uninodular tumor & $55(19.64)$ & & & $89(16.21)$ & & & $144(17.37)$ & & \\
\hline Multinodular tumor & $107(38.21)$ & & & $228(41.53)$ & & & $335(40.41)$ & & \\
\hline Massive tumor & $92(32.86)$ & & & $168(30.60)$ & & & $260(31.36)$ & & \\
\hline Missing & $7(2.50)$ & & & $21(3.83)$ & & & $28(3.38)$ & & \\
\hline \multicolumn{10}{|l|}{ Differentiation } \\
\hline Well & $31(11.07)$ & & & $77(14.03)$ & & & $108(13.03)$ & & \\
\hline Moderate & $78(27.86)$ & & & $195(35.52)$ & & & $273(32.93)$ & & \\
\hline Poor & $171(61.07)$ & & & $277(50.46)$ & & & $448(54.04)$ & & \\
\hline \multicolumn{10}{|l|}{ Metastasis } \\
\hline Abscent & $81(28.93)$ & & & $189(34.43)$ & & & $270(32.57)$ & & \\
\hline Present & $193(68.93)$ & & & $347(63.21)$ & & & $540(65.14)$ & & \\
\hline Missing & $6(2.14)$ & & & $13(2.37)$ & & & $19(2.29)$ & & \\
\hline \multicolumn{10}{|l|}{ TNM stage } \\
\hline । & $53(18.93)$ & & & $148(26.96)$ & & & $201(24.25)$ & & \\
\hline$\|$ & $95(33.93)$ & & & $230(41.89)$ & & & $325(39.20)$ & & \\
\hline III & $64(22.86)$ & & & $110(20.04)$ & & & $174(20.99)$ & & \\
\hline IV & 68 (24.29) & & & $61(11.11)$ & & & $129(15.56)$ & & \\
\hline
\end{tabular}

$F$ females, $M$ males, $S D$ standard deviation, AFP alpha fetoprotein, $T N M$ tumor, nodes, metastasis-classification ${ }^{a}$ Kruskal-Wallis test for continuous variables

${ }^{\mathrm{b}} \mathrm{Chi}$ square test for categorical variables

were used to redistribute controls and patients. By convention if $P<0.05$, the difference was considered statistically significant.
A Q-Q plot was then graphed to check the $P$ value distribution. The 'cumulative distribution function' of the normal density and qth quantile of a Gauss distribution 
was signified by $\Phi(\mathrm{z})$ and $\xi_{\mathrm{q}}$, respectively, $\left(\Phi\left(\xi_{\mathrm{q}}\right)=\mathrm{q}\right)$. Therefore, the probability $<\xi q$ is actually just q. The theoretical quantile was defined by the inverse of the normal cumulative distribution function. Especially, the theoretical fitting the empirical quantile $\mathrm{z}_{(\mathrm{i})}$ should be

$$
\begin{aligned}
& \xi_{q}=q \approx \frac{i-0.5}{n}, \\
& \text { for } i=1,2,3, \ldots, \mathrm{n} .
\end{aligned}
$$

\section{SNP selection and TaqMan genotyping in the following replication study}

SNPs in CHD5 were selected on the basis of 'significant SNPs' found in the discovery-targeted NGS results of 255 controls and 280 HCC samples. Next, genomic DNAs from all other subjects (510 controls and 549 patients) were genotyped using TaqMan probes with the ABI 7500 Fast System (Applied Biosystems, forster City, CA) for the selected two SNPs in haplotypic block 3 (rs12564469 and rs9434711). PCRs were performed with 50 ng DNA in 25-ul total volume containing $0.25 \mathrm{ul} \mathrm{Taq}$ polymerase, $2.5 \mathrm{ul} \mathrm{PCR} \mathrm{mix,} 0.625 \mathrm{ul}$ of each primer and $2.5 \mathrm{ul} \mathrm{dNTPs}$ for 40 cycles of denaturation $\left(95^{\circ} \mathrm{C}\right)$ for 10 min, annealing $\left(92{ }^{\circ} \mathrm{C}\right)$ for $15 \mathrm{~s}$ and extension $\left(60{ }^{\circ} \mathrm{C}\right)$ for $1 \mathrm{~min}$. Associations of the potential risk SNPs or haplotypes with HCC were further evaluated by stratification analysis with subgroups of age, sex, smoking and drinking status. Pi was defined as the division of the two $P$ numbers, which means the larger in absolute terms indicating more meaningful value.

\section{False-positive report probability (FPRP) analysis}

To avoid the possibility of false-positives inherent to performing multiple tests, a Bayesian statistical test-the FPRP-was performed for all significance in genetic association studies [23]. According to the method proposed, an FPRP value of $\leq 0.2$ was regarded as pointing to a significant association, and a prior probability of 0.1 to check ORs of 1.50/0.67 was applied for risk/protective functions. The statistical power was calculated according to the case/control numbers and $\mathrm{OR} / P$ values in the study.

\section{Gene-environment interactions}

The possible gene-environment interactions with high-order in the associations were evaluated using the multiple dimension reduction (MDR) programme [24]. Briefly, we carried out a 100-fold cross-validation and 1000 -fold permutations under the assumption of no association. The maximum cross-validation consistency $(\mathrm{CVC})$ and minimum average prediction error were requirements for the best interaction model.

\section{Statistical software}

The SPSS 22.0 for Windows (SPSS, Chicago, IL) and R scripts (3.0.2 Suite) software were used for statistical analyses.

\section{Results}

Population association risk (PAR) in the discovery study We detected a total of 164 single-base substitutions analysing the targeted NGS results (Fig. 1a and Additional file 1: Table S1). Of these, eight were in a promoter region, 129 were intronic and 27 were in coding exons. A case-control study was conducted and the results indicated potential associations between the risk of $\mathrm{HCC}$ and the CHD5 polymorphisms rs9434741 (PAR = $0.0051)$, rs2273032 (PAR $=0.0089)$ and rs12067480 (PAR $=0.0261$ ) in the Han population (Fig. $1 \mathrm{~b}$ and Additional file 1: Table S1). But they lost statistical significance after performing a Bonferroni correction. They also lost their significance after $10^{5}$ permutation tests (for example, $P$ $=0.3156$ for rs9434741, Fig. 1c). Q-Q plots were used to compare with the observed chi-square results with the distribution expected under the null hypothesis, there was deviation from expectation at a higher value of approximately 2.8 (Fig. 1d). After removing rs9434741, there were no significant curve changes compared with the expected distribution (Fig. 1e).

\section{LD and haplotypic analysis in the discovery study}

Direct sequencing results revealed a total of 164. SNPs in CHD5. We identified three blocks with high LD (Fig. 1a). Block 1 includes SNP3-SNP6 (rs12037962, rs11587, rs41307753 and rs3810989). Block 2 includes SNP35SNP38 (rs2273041, rs2273040, rs2273038 and rs55930553). Block 3 includes SNP115 and SNP116 (rs12564469 and rs9434711). Blocks were reconstructed according to their frequencies. The results of the haplotype-based case-control study between the HCC and control groups are shown in Table 2. We found that a haplotype AG in block 3 showed a significant association with HCC $\left(P=1.94 \times 10^{-5}\right)$. It remained significant according to unconditional logistic regression analysis after adjustment for age, sex, smoking and drinking status $\left(P_{\text {corrected }}=5.73 \times 10^{-5}\right)$ and after $10^{5}$ permutation tests $\left(P=4.00 \times 10^{-5}\right)$.

Population association and haplotypic analysis based on selected SNPs in the replication and combined studies We selected SNPs rs12564469 and rs9434711 in block 3 from the first SNP discovery study for the next study. Replicative results showed no associations for rs12564469 $\left(\mathrm{PAR}=0.0800, P_{\text {adjusted }}=0.1029, P_{\text {Permutation }}=0.1062\right)$ or for rs9434711 $\left(\mathrm{PAR}=0.8718, P_{\text {adjusted }}=0.8485, P_{\text {Permutation }}\right.$ $=0.9601)$. Finally, a combined study including discovery and replicative cohort data was conducted. Combined 


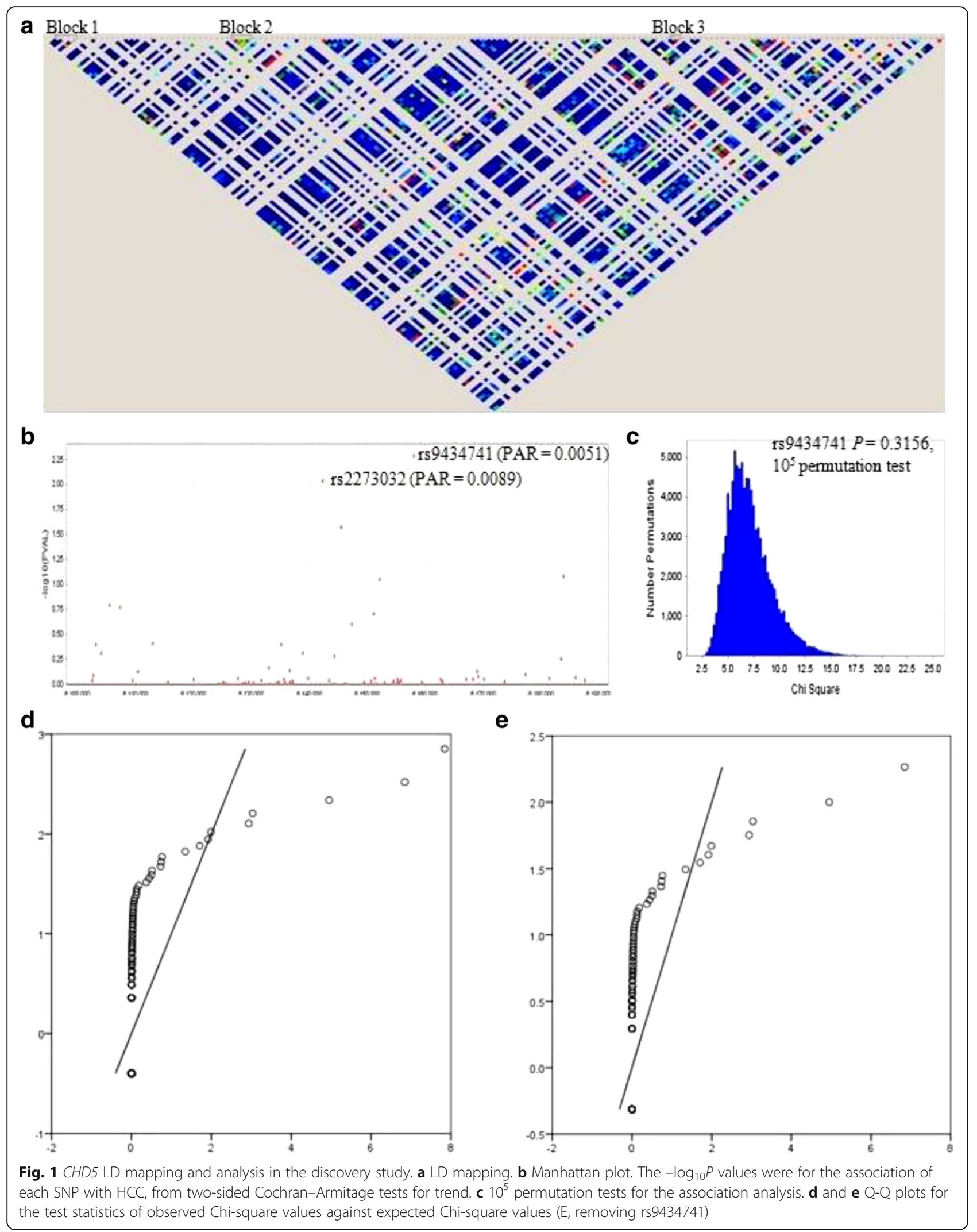


Table 2 Haplotype frequencies in the discovery, replication and combined studies

\begin{tabular}{|c|c|c|c|c|c|c|}
\hline Haplotypes & Case, Control Ratio Counts ${ }^{\mathrm{a}}$ & Case, Control Frequencies $^{\mathrm{b}}$ & Chi Square & PAR & $P_{\text {corrected }}{ }^{c}$ & $P_{\text {Permutation }}{ }^{d}$ \\
\hline \multicolumn{7}{|c|}{ Discovery study } \\
\hline \multicolumn{7}{|l|}{ Block 1} \\
\hline GGCA & 176.0: 384.0, 171.1: 338.9 & $0.314,0.335$ & 0.54 & 0.4623 & 0.2970 & 0.9976 \\
\hline GACA & 176.0: 384.0, 171.1: 338.9 & $0.314,0.335$ & 0.54 & 0.4623 & 0.2970 & 0.9976 \\
\hline AGCG & 67.5: 492.5, 61.1: 448.9 & $0.121,0.120$ & 0.002 & 0.9651 & 0.8263 & 1 \\
\hline AACG & $67.5: 492.5,61.1: 448.9$ & $0.121,0.120$ & 0.002 & 0.9651 & 0.8263 & 1 \\
\hline AGCA & 29.5: 530.5, 20.9: 489.1 & $0.053,0.041$ & 0.795 & 0.3727 & 0.6037 & 0.9876 \\
\hline AACA & 29.5: 530.5, 20.9: 489.1 & $0.053,0.041$ & 0.795 & 0.3727 & 0.6037 & 0.9876 \\
\hline \multicolumn{7}{|l|}{ Block 2} \\
\hline CCCG & 417.3: 126.7, 375.9: 118.1 & $0.767,0.761$ & 0.055 & 0.8153 & 0.7749 & 1 \\
\hline TाTA & 42.0: 502.0, 37.3: 456.7 & $0.077,0.076$ & 0.011 & 0.9171 & 0.9690 & 1 \\
\hline CCTG & 36.0: 508.0, 35.5: 458.5 & $0.066,0.072$ & 0.128 & 0.7208 & 0.8452 & 1 \\
\hline TTCA & 25.5: 518.5, 25.2: 468.8 & $0.047,0.051$ & 0.098 & 0.7544 & 0.7805 & 1 \\
\hline CCCA & 9.5: $534.5,8.4: 485.6$ & $0.017,0.017$ & 0.002 & 0.9622 & 0.9417 & 1 \\
\hline TाTG & 7.7: 536.3, 5.8: 488.2 & $0.014,0.012$ & 0.121 & 0.7282 & 0.7548 & 1 \\
\hline \multicolumn{7}{|l|}{ Block 3} \\
\hline AA & 324.0: 170.0, 289.0: 151.0 & $0.656,0.657$ & 0.001 & 0.9757 & 0.8983 & 1 \\
\hline GG & 143.8: 350.2, 148.8: 291.2 & $0.291,0.338$ & 2.399 & 0.1214 & 0.1665 & 0.5747 \\
\hline$A G$ & $26.2: 467.8,2.2: 437.8$ & $0.053,0.005$ & 18.248 & $1.94 \times 10^{-5}$ & $5.73 \times 10^{-5}$ & $4.00 \times 10^{-5}$ \\
\hline \multicolumn{7}{|c|}{ Replication study } \\
\hline \multicolumn{7}{|l|}{ Block 3} \\
\hline $\mathrm{AA}$ & 630.9: 341.1, 579.0: 309.0 & $0.649,0.652$ & 0.018 & 0.8945 & $0.8714^{8}$ & 0.9893 \\
\hline GG & 294.5: 677.5, 303.5: 584.5 & $0.303,0.342$ & 3.202 & 0.0735 & 0.1069 & 0.1542 \\
\hline$A G$ & 46.6: 925.4, 5.5: 882.5 & $0.048,0.006$ & 29.716 & $5.038 \times 10-^{8}$ & $7.571 \times 10-$ & 0.00001 \\
\hline \multicolumn{7}{|c|}{ Combined study } \\
\hline \multicolumn{7}{|l|}{ Block 3} \\
\hline AA & 954.9: 511.1, 868.0: 460.0 & $0.651,0.654$ & 0.015 & 0.9012 & 0.9467 & 0.9909 \\
\hline GG & 438.3: 1027.7, 452.3:875.7 & $0.299,0.341$ & 5.556 & 0.0184 & 0.0383 & 0.0410 \\
\hline$A G$ & 72.7: 1393.3, 7.7: 1320.3 & $0.050,0.006$ & 47.941 & $4.393 \times 10^{-12}$ & $5.514 \times 10^{-11}$ & 0.00001 \\
\hline
\end{tabular}

Block 1, rs12037962, rs11587, rs41307753 and rs3810989

Block 2, rs2273041, rs2273040, rs2273038 and rs55930553

Block 3, rs 12564469 and rs9434711

${ }^{a}$ Number of haplotypes were compared in cases versus controls: Haplotype(1):haplotype(others) cases, Haplotype(1):haplotype(others) controls

${ }^{\mathrm{b}}$ Frequency of the haplotype

'Calculated in logistical regression models with adjustment for age, gender, smoking and drinking status; $p<0.005$ means significant value by Bonferroni

correction based on the total number of markers genotyped

${ }^{\mathrm{d} E m p i r i c a l} p$-value based on $10^{5}$ permutations of case-control status using the $\max (\mathrm{T})$ procedure. $p<0.05$ means significant value

results also showed no association for rs12564469 (PAR = $\left.0.0210, \quad P_{\text {adjusted }}=0.0290, \quad P_{\text {Permutation }}=0.0286\right)$ and for rs9434711 (PAR $=0.8829, P_{\text {adjusted }}=0.9137, P_{\text {Permutation }}=$ 0.9704; Table 3).

The results of the haplotype-based replication and combined studies between the HCC and control groups are shown in Table 2. We observed increased frequencies of haplotype AG in $\mathrm{HCC}$ patients compared with those seen in healthy controls both in the replication study (PAR = $\left.5.038 \times 10^{-8}, P_{\text {adjusted }}=7.571 \times 10^{-8}, P_{\text {Permutation }}=0.00001\right)$ and in the combined study $\left(\mathrm{PAR}=4.393 \times 10^{-12}, P_{\text {adjusted }}=\right.$ $\left.5.514 \times 10^{-11}, P_{\text {Permutation }}=0.00001\right)$.

\section{Stratification analysis of haplotypes}

The association of haplotype AG (block 3) with the risk of HCC in subgroups such as age, sex, smokers and drinkers were evaluated further using replication and combined studies (Table 4). We found that those individuals carrying haplotype AG had a significantly increased risk of $\mathrm{HCC}$, and the risk was 
Table 3 rs 12564469 and rs9434711 in replication and combined studies

\begin{tabular}{|c|c|c|c|c|c|c|c|}
\hline & Alleles $^{a}$ & Case, Control Ratio Counts ${ }^{b}$ & Case, Control Frequencies $^{c}$ & Chi square & PAR $^{d}$ & $P_{\text {adjusted }}{ }^{e}$ & $P_{\text {permutation }}^{f}$ \\
\hline \multicolumn{8}{|l|}{ Replication } \\
\hline rs12564469 & $A>G$ & 659:289, 570:298 & $0.695,0.657$ & 3.065 & 0.0800 & 0.1029 & 0.1062 \\
\hline rs9434711 & $A>G$ & $341: 629,309: 579$ & $0.352,0.348$ & 0.026 & 0.8718 & 0.8485 & 0.9601 \\
\hline \multicolumn{8}{|l|}{ Combined } \\
\hline rs12564469 & $A>G$ & $1003: 431,857: 445$ & $0.699,0.658$ & 5.328 & 0.0210 & 0.0290 & 0.0286 \\
\hline rs9434711 & $A>G$ & $511: 953,460: 868$ & $0.349,0.346$ & 0.022 & 0.8829 & 0.9137 & 0.9704 \\
\hline
\end{tabular}

${ }^{\text {a }}$ The major allele is listed first, then the minor allele

${ }^{b}$ Number of alleles were compared in cases versus controls: allele(1):allele(2) cases, allele(1):allele(2) controls

cFrequency of the association allele

${ }^{\mathrm{d} P A R}$, population attributable risk

${ }^{e}$ Calculated in logistical regression models with adjustment for age, gender, smoking and drinking status

${ }^{\mathrm{f}} P$ for $10^{5}$ permutation test

increased in patients of $>55$ years $\left(P=6.04 \times 10^{-8}\right.$ and $\left.P_{\mathrm{i}} \quad(\mathrm{P} 2 / \mathrm{P} 1)=5.12 \times 10^{-4}\right)$ and in drinkers $(P=$ $9.43 \times 10^{-8}$ and $\left.P_{\mathrm{i}(\mathrm{P} 2 / \mathrm{P} 1)}=3.25 \times 10^{-6}\right)$.

\section{FPRP}

The significant associations of FPRP values for block 3 haplotype AG (vs. AA + GG) at different levels of prior probability are listed in Table 5. FPRP values of haplotype AG for $\mathrm{HCC}$ risk in patients $>55$ years were $<0.20$ for the assigned prior probability $(0.017$ for the prior probability of 0.1 in the replication study; 0.004 and 0.010 for the prior probabilities of 0.1 and 0.01 , respectively, in the combined study). For the risk of HCC in alcohol drinkers, when the assumptions of prior probability were 0.1 and 0.01 , all findings were significant not only in the discovery study but also in the replication and combined studies (FPRP <0.20). Moreover, when the assumption of prior probability was 0.001 , this association was still prominent in the combined study $($ FPRP $=0.069)$.

\section{Association of high-order interactions with HCC risk by MDR}

The interactions of high-order assessed with MDR were conducted, including the potential risk haplotype AG and four known risk factors (age, sex, smoking and drinking status), in order to check whether possible gene-environmental interactions in association with the risk of HCC exists. In the discovery study, we noticed that the best one-factor model was drinking status, with the highest CVC (99/100, the same model is selected as the best model 99 out of 100 times) and the lowest prediction error (0.385). The best model for two-factors was drinking status plus haplotype AG, with the highest CVC (96/100) and the lowest prediction error (0.403). Interestingly, the model with 5 -factors had a maximum CVC (100/100) and a minimum prediction error (0.378). This is a model with better prediction than the model with one factor. Same results were found in the replication study and the combined study (Table 6).

\section{Discussion}

Studies have found that the chromosome aberration of 1p36 deletion is not frequent in HCC. It remains to be determined whether the common SNPs in CHD5 are associated with the risk of HCC. CHD5 is a tumour-suppressing gene of the chromodomain gene family, first identified as a tumour-suppressing gene mapping to 1 p36.31 [25].

The integration of clinical phenotypes and genomic information may enable precision cancer medicine through NGS approaches [26]. Results of our targeted NGS and TaqMan genotyping revealed no significant associations with the risk of HCC neither in the discovery study nor in the replication and combined studies. For two data sets, it is important to identify whether the hypothesis of a common distribution is proven to be true. The Q-Q plot offers more insight into the discrepancy than any other statistical analysis such as the Kolmogorov Smirnov 2-sample test or the chi-square test. However, we did not find any significant change after removing rs9434741, which suggests that the most likely associated SNP is not a risk locus.

Nonetheless, we inadvertently found a positive association of a rare haplotype AG (block 3: rs-12564469-rs9434711) in CHD5 and HCC, which has not been reported to date. Importantly, this association quality increased in a gene dose-dependent manner as the number of samples increased (PAR in Table 2). Thus, our results support the idea that the 1p36 region plays a role in HCC. We believe it is possible that hereditary mutations of tumour-suppressing genes in the 1 p36 region contribute to the aggressive properties of liver cancer. Hereditary changes in the $1 \mathrm{p} 36$ region are extraordinarily common in human tumours, occurring in malignancies of epithelial, neural and haematopoietic origin [25]. Genetic mutations of the tumour-suppressing gene CHD5 have conduced to the understanding of human oncogenesis. 


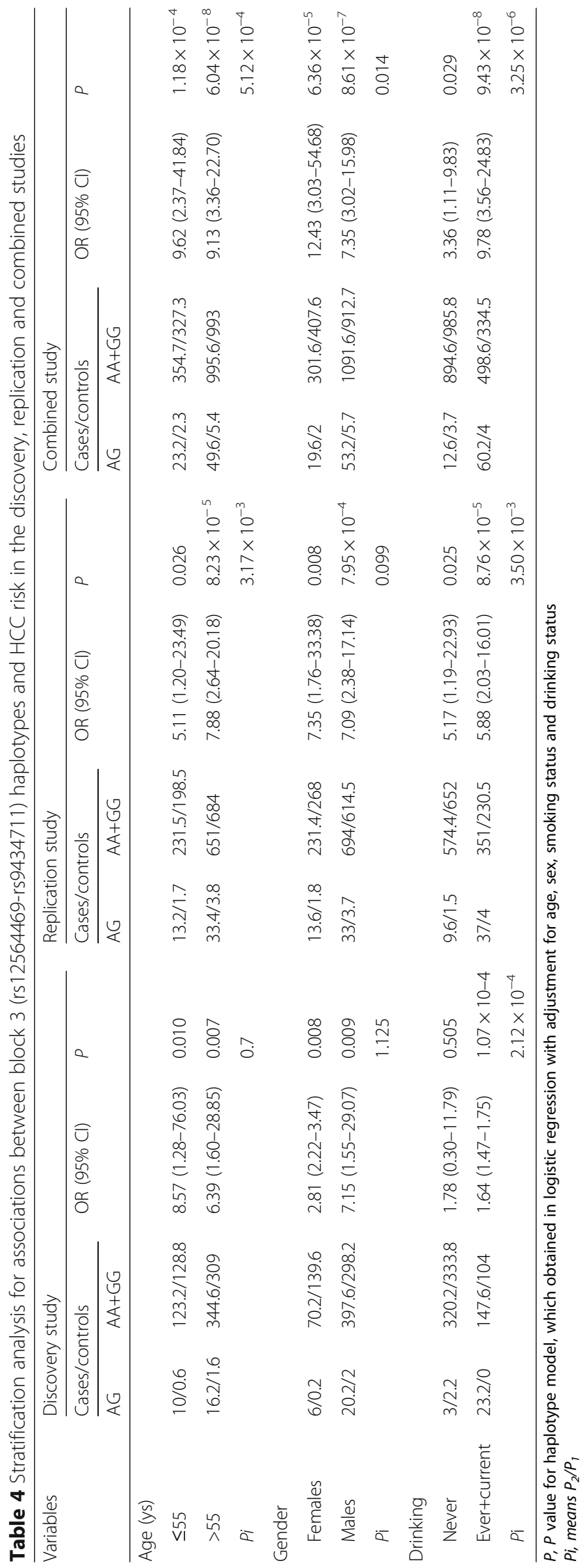


Table 5 FPRP values for associations between HCC risk and block 3 haplotype frequencies ( $A G$ vs. AA+GG)

\begin{tabular}{lcccccc}
\hline Variables & $\begin{array}{c}\text { Statistical } \\
\text { power }^{\mathrm{a}}\end{array}$ & \multicolumn{4}{l}{ Prior probability } \\
\cline { 5 - 7 } & 0.1 & 0.01 & 0.001 & 0.0001 \\
\hline HCC risk in >55 years old group & & & & \\
\multicolumn{2}{l}{ Discovery study } & 0.704 & 0.216 & 0.493 & 0.721 & 0.885 \\
Replication study & 0.689 & 0.017 & 0.271 & 0.525 & 0.843 \\
Combined study & 0.837 & 0.004 & 0.010 & 0.347 & 0.706 \\
HCC risk in drinking group & & & & \\
Discovery study & 0.792 & 0.003 & 0.013 & 0.298 & 0.635 \\
Replication study & 0.658 & 0.005 & 0.017 & 0.424 & 0.757 \\
Combined study & 1 & $<0.001$ & 0.005 & 0.069 & 0.236 \\
\hline
\end{tabular}

Block 3, rs12564469 and rs9434711

If the prior probability $<0.20$, the results in FPRP are in bold

It seems that the risk effect of the haplotype AG was more evident in the drinkers' subgroup (Ref: $P_{\mathrm{i}}$ in Table 4) with the stratified analysis. One of the possible comments is that the sample size is smaller in subgroups. Nevertheless, the results of the FPRP analysis for those findings showed that the drinkers group remained significant at the prior probability level of 0.1 . We believe that in drinkers,

Table 6 MDR analysis for the prediction of HCC risk with and without haplotype AG

\begin{tabular}{|c|c|c|c|}
\hline $\begin{array}{l}\text { Best interaction } \\
\text { models }\end{array}$ & Cross-validation & $\begin{array}{l}\text { Average prediction } \\
\text { error }\end{array}$ & $p^{a}$ \\
\hline \multicolumn{4}{|l|}{ Distcovery study } \\
\hline 1 & $99 / 100$ & 0.385 & $<0.0001$ \\
\hline 1,2 & $96 / 100$ & 0.403 & $<0.0001$ \\
\hline $1,2,3$ & $100 / 100$ & 0.401 & $<0.0001$ \\
\hline $1,2,3,4$ & $87 / 100$ & 0.380 & $<0.0001$ \\
\hline $1,2,3,4,5^{b}$ & $100 / 100$ & 0.378 & $<0.0001$ \\
\hline \multicolumn{4}{|l|}{ Replication study } \\
\hline 1 & $95 / 100$ & 0.412 & $<0.0001$ \\
\hline 1,2 & $94 / 100$ & 0.417 & $<0.0001$ \\
\hline $1,2,3$ & $98 / 100$ & 0.389 & $<0.0001$ \\
\hline $1,2,3,4$ & $90 / 100$ & 0.383 & $<0.0001$ \\
\hline $1,2,3,4,5^{b}$ & $100 / 100$ & 0.368 & $<0.0001$ \\
\hline \multicolumn{4}{|l|}{ Combined study } \\
\hline 1 & $96 / 100$ & 0.399 & $<0.0001$ \\
\hline 1,2 & $94 / 100$ & 0.410 & $<0.0001$ \\
\hline $1,2,3$ & $99 / 100$ & 0.396 & $<0.0001$ \\
\hline $1,2,3,4$ & $89 / 100$ & 0.382 & $<0.0001$ \\
\hline $1,2,3,4,5^{b}$ & $100 / 100$ & 0.375 & $<0.0001$ \\
\hline
\end{tabular}

Labels: 1, drinking status; 2, haplotype AG (block 3); 3, age; 4, smoking status; 5 , gender

${ }^{a} P$ value for 1000 -fold permutation test

${ }^{\mathrm{b}}$ The best model with maximum cross-validation consistency and minimum prediction error rate alcohol-related carcinogens may cause DNA damage [27] and that accumulated DNA damage caused by the regular carcinogenic exposure to alcoholic drinks $[28,29]$ might enhance the effect of genetic instability.

Next, we conducted a high-order gene (haplotype)-environment interaction analysis with MDR testing to support the above results. The best interaction model revealed that the CHD5 haplotype AG interacted with the drinking status with a maximal $\mathrm{CVC}$ and minimal prediction error, which was more obvious in the interaction entropy analysis. Our results suggested that the stratification testing reliably identified alcohol drinking as a risk factor.

Our recent study had reported that the CHD5 rs12564469-rs9434711 region might functionally contribute to HCC prognosis and CHD5 mRNA expressions [30]. It is possible that CHD5 plays an essential role in cancer development. The expression of multiple genes that regulate pathways in the tumourigenic process was modulated by CHD5 [31]. Apoptosis, cellular senescence and neonatal death will occur by excessive activation of these tumour-suppressive pathways, dependent on $\mathrm{p} 53$, p19 and p16. CHD5 expression seems to be restricted to neural-derived tissues, as opposed to CHD4 which is expressed in all tissues. CHD5 mRNA cannot be detected in the liver, placenta, spleen, bone marrow, thyroid, stomach, pancreas, small intestine, colon or prostate $[8,30]$. Because of this, expression of the candidate tumour-suppressing genes was sequentially disrupted by specific shRNAs. What is more, CHD5 expression is down-regulated in HCC tissues and HepG2, and the expression level of CHD5 was inversely correlated with the expression of oncogene miR-454 in HCC tissues [32]. Therefore, CHD5 as the cause of the observed phenotype was identified.

Alternatively, CHD5 or a CHD5-containing complex could interact with p53 directly. A similar model for a MTA2-containing NuRD complex regulating the p53-mediated transactivation by modulating the p53 acetylation status [33] was suggested. CHD5 may function in a similar manner since it was shown to be part of a NuRD-like complex [34]. Both the interactions and functions are equally important for the development of HCC. The genetic engineering mice with a heterozygous deficiency of the (human) 1p36 locus were prone to develop non-neural tumours (lymphoma, squamous cell carcinoma and hibernoma). CHD5 was found to positively regulate p53 presumably via p14/p19ARF [35, 36]. But the exact molecular mechanisms could not be defined. 


\section{Conclusions}

In short, we identified a rare haplotype in CHD5 that was significant associated with the risk of HCC. Our results highlight the breadth of precision medicine by providing clues to help the advancement of effective diagnostic, management and prevention tools against cancer. Nonetheless, larger sample size studies are needed to corroborate our findings.

\section{Additional file}

Additional file 1: Table S1. A case-control study in the discovery study with targeted NGS. (XLS $37 \mathrm{~kb}$ )

\section{Abbreviations}

CHD5: Chromodomain helicase DNA-binding protein 5; FPRP: False-positive report probability Population risk evaluation; HCC: Hepatocellular carcinoma; LD: Linkage disequilibrium; MDR: Multiple dimension reduction; NGS: Targeted next-generation sequencing; Q-Q: Permutation test and quantile-quantile; SNPs: Single-nucleotide polymorphisms

\section{Acknowledgments}

We would like to thank all the participants that contribute to this work. The authors would like to thank Enago (www.enago.cn) for the English language review.

\section{Funding}

Supported by Guangdong Provincial Science and Technology Programs (2014A020212653 and 2016A050503046); The Public Service Platform of South China Sea for R\&D Marine Biomedicine Resources (2018008), and Science and Technology Research Project in Dongguan City (2014108101048).

\section{Availability of data and materials}

The datasets generate and analyzed in this study are not publicly available for the reason of protecting patients' privacy, but are available from the corresponding author (TT) on reasonable request.

\section{Authors' contributions}

TT, XZ and HL designed the study. LC, QX, HaiL, HLi, MZ, FL and XZ analyzed the patient data and carried out the genotyping. QK, FJ and SP performed the statistical analyzes. XZ, WF and TT wrote the manuscript. LC, HaiL, MZ and $\Pi$ contributed samples and patient information. All authors read and approved the final draft of the manuscript.

\section{Ethics approval and consent to participate}

The ethics committee of Guangdong Medical University authorised the experimental and research protocols of this study. All procedures performed in studies were in accordance with the ethical standards of the institutional and/or national research committee and with the 1964 Helsinki declaration and its later amendments or comparable ethical standards. All controls and and patients (or relatives of patients who already died) provided written informed consent.

\section{Competing interests}

The authors declare that they have no competing interests.

\section{Publisher's Note}

Springer Nature remains neutral with regard to jurisdictional claims in published maps and institutional affiliations.

\section{Author details}

${ }^{1}$ Guangdong Provincial Key Laboratory of Medical Molecular Diagnostics, Dongguan Scientific Research Center, Guangdong Medical University, Dongquan, China. ${ }^{2}$ Department of Blood Transfusion, Peking University Shenzhen Hospital, Shenzhen, China. ${ }^{3}$ Digestive System Tumor Tissue Bank,
Center of Surgery Laboratory, The First Affiliated Hospital, Sun Yat-sen University, Guangzhou, China. ${ }^{4}$ The Affiliated Hospital Cancer Center, Guangdong Medical University, Zhanjiang, China. ${ }^{5}$ Department of Pathology, Guangdong Medical University, Dongguan, China. ${ }^{6}$ Immunity and Biochemical Research Lab, Zhejiang Academy of Medical Sciences, Hangzhou, China. ${ }^{7}$ Department of Biochemistry and Molecular Biology, Binzhou Medical University, Yantai, China. ${ }^{8}$ Department of Gastroenterology, Zibo Central Hospital, Zibo, China. ${ }^{9}$ Forensic Identification Institute, Guangdong Women and Children Hospital, Guangzhou, China. ${ }^{10}$ Guanghua School of Stomatology, Hospital of Stomatology, Sun Yat-sen University, Guangzhou, China.

Received: 31 December 2017 Accepted: 24 May 2018 Published online: 15 June 2018

\section{References}

1. Zhang Y, Liu H, Yi R, Yan T, He Y, Zhao Y, Liu J. Hepatitis B virus whole-X and $X$ protein play distinct roles in HBV-related hepatocellular carcinoma progression. J Exp Clin Cancer Res. 2016;35(1):87.

2. Baykara O, Tansarikaya M, Bulut P, Demirkaya A, Buyru N. CHD5 is a potential tumor suppressor in non small cell lung cancer (NSCLC). Gene. 2017:618:65-8.

3. Higashi M, Kolla V, lyer R, Naraparaju K, Zhuang T, Kolla S, Brodeur GM. Retinoic acid-induced CHD5 upregulation and neuronal differentiation of neuroblastoma. Mol Cancer. 2015;14:150.

4. Lang J, Tobias ES, Mackie R. Preliminary evidence for involvement of the tumour suppressor gene CHD5 in a family with cutaneous melanoma. $\mathrm{Br}$ J Dermatol. 2011;164(5):1010-6.

5. Zhao R, Yan Q, Lv J, Huang H, Zheng W, Zhang B, Ma W. CHD5, a tumor suppressor that is epigenetically silenced in lung cancer. Lung Cancer. 2012; 76(3):324-31.

6. Wu X, Zhu Z, Li W, Fu X, Su D, Fu L, Zhang Z, Luo A, Sun X, Fu L, et al. Chromodomain helicase DNA binding protein 5 plays a tumor suppressor role in human breast cancer. Breast Cancer Res. 2012;14(3):R73.

7. Wong RR, Chan LK, Tsang TP, Lee CW, Cheung TH, Yim SF, Siu NS, Lee SN, Yu MY, Chim SS, et al. CHD5 downregulation associated with poor prognosis in epithelial ovarian cancer. Gynecol Obstet Investig. 2011; 72(3):203-7

8. Xu G, Zhu H, Zhang M, Xu J. Histone deacetylase 3 is associated with gastric cancer cell growth via the miR-454-mediated targeting of CHD5. Int J Mol Med. 2018;41(1):155-63.

9. Fatemi M, Paul TA, Brodeur GM, Shokrani B, Brim H, Ashktorab H. Epigenetic silencing of CHD5, a novel tumor-suppressor gene, occurs in early colorectal cancer stages. Cancer. 2014;120(2):172-80.

10. Fang $Q L$, Yin YR, Xie CR, Zhang S, Zhao WX, Pan C, Wang XM, Yin ZY. Mechanistic and biological significance of DNA methyltransferase 1 upregulated by growth factors in human hepatocellular carcinoma. Int J Oncol. 2015:46(2):782-90.

11. Zhu X, Zhang J, Fan W, Wang F, Yao H, Wang Z, Hou S, Tian Y, Fu W, Xie D, et al. The rs391957 variant cis-regulating oncogene GRP78 expression contributes to the risk of hepatocellular carcinoma. Carcinogenesis. 2013; 34(6):1273-80.

12. Sangiovanni A, Colombo M. Treatment of hepatocellular carcinoma: beyond international guidelines. Liver Int. 2016:36(Suppl 1):124-9.

13. Braillon A. Recurrence in early-stage hepatocellular carcinoma: CpG methylation and smoking. J Clin Oncol. 2017;35(18):2097-8.

14. Tarabichi Y, Kats DJ, Kaelber DC, Thornton JD. The impact of fluctuations in pack-year smoking history in the electronic health record on lung cancer screening practices. Chest. 2018;153(2):575-8.

15. Offermans NSM, Ketcham SM, van den Brandt PA, Weijenberg MP, Simons C. Alcohol intake, $\mathrm{ADH1B}$ and $\mathrm{ADH} 1 \mathrm{C}$ genotypes, and the risk of colorectal cancer by sex and subsite in the Netherlands Cohort Study. Carcinogenesis. 2018;39(3):375-88

16. Kiialainen A, Karlberg O, Ahlford A, Sigurdsson S, Lindblad-Toh K, Syvanen AC. Performance of microarray and liquid based capture methods for target enrichment for massively parallel sequencing and SNP discovery. PLoS One. 2011;6(2):e16486.

17. Garcia-Seco D, Zhang Y, Gutierrez-Manero FJ, Martin C, Ramos-Solano B. RNA-Seq analysis and transcriptome assembly for blackberry (Rubus sp. Var. Lochness) fruit. BMC Genomics. 2015;16:5. 
18. Perkins BA, Caskey CT, Brar P, Dec E, Karow DS, Kahn AM, Hou YC, Shah N, Boeldt D, Coughlin E, et al. Precision medicine screening using wholegenome sequencing and advanced imaging to identify disease risk in adults. Proc Natl Acad Sci U S A. 2018:115(14):3686-91.

19. Purcell S, Neale B, Todd-Brown K, Thomas L, Ferreira MA, Bender D, Maller J, Sklar P, de Bakker PI, Daly MJ, et al. PLINK: a tool set for whole-genome association and population-based linkage analyses. Am J Hum Genet. 2007; 81(3):559-75.

20. Smith AV. Manipulating HapMap Data Using HaploView. CSH Protoc. 2008; 2008:pdb prot5025.

21. Dai JY, Ruczinski I, LeBlanc M, Kooperberg C. Imputation methods to improve inference in SNP association studies. Genet Epidemiol. 2006;30(8): 690-702.

22. Long JC, Williams RC, Urbanek M. An E-M algorithm and testing strategy for multiple-locus haplotypes. Am J Hum Genet. 1995;56(3):799-810.

23. Obazee O, Capurso G, Tavano F, Archibugi L, De Bonis A, Greenhalf W, Key T, Pasquali C, Milanetto AC, Hackert T, et al. Common genetic variants associated with pancreatic adenocarcinoma may also modify risk of pancreatic neuroendocrine neoplasms. Carcinogenesis. 2018;39(3):360-7.

24. Moore $\mathrm{JH}$, Andrews PC. Epistasis analysis using multifactor dimensionality reduction. Methods Mol Biol. 2015;1253:301-14.

25. Henrich KO, Schwab M, Westermann F. 1 p36 tumor suppression-a matter of dosage? Cancer Res. 2012;72(23):6079-88.

26. Gagan J, Van Allen EM. Next-generation sequencing to guide cancer therapy. Genome Med. 2015;7(1):80

27. Cederbaum Al, Lu Y, Wu D. Role of oxidative stress in alcohol-induced liver injury. Arch Toxicol. 2009;83(6):519-48.

28. Xie Z, Tan G, Ding M, Dong D, Chen T, Meng X, Huang X, Tan Y. Foxm1 transcription factor is required for maintenance of pluripotency of P19 embryonal carcinoma cells. Nucleic Acids Res. 2010;38(22):8027-38.

29. El Idrissi M, Hervieu V, Merle P, Mortreux F, Wattel E. Cause-specific telomere factors deregulation in hepatocellular carcinoma. J Exp Clin Cancer Res. 2013:32:64.

30. Zhu X, Kong Q, Xie L, Chen Z, Li H, Zhu Z, Huang Y, Lan F, Luo H, Zhan J, et al. The single-nucleotide polymorphisms in CHD5 affect the prognosis of patients with hepatocellular carcinoma. Oncotarget. 2018;9(17):13222-30

31. Tamura S, Wang Y, Veeneman B, Hovelson D, Bankhead A 3rd, Broses $L$, Lorenzatti Hiles G, Liebert M, Rubin JR, Day KC, et al. Molecular correlates of in vitro responses to dacomitinib and afatinib in bladder cancer. Bladder Cancer. 2018;4(1):77-90.

32. Yu L, Gong X, Sun L, Yao H, Lu B, Zhu L. miR-454 functions as an oncogene by inhibiting CHD5 in hepatocellular carcinoma. Oncotarget. 2015;6(36): 39225-34.

33. Luo J, Su F, Chen D, Shiloh A, Gu W. Deacetylation of p53 modulates its effect on cell growth and apoptosis. Nature. 2000;408(6810):377-81.

34. Kolla V, Naraparaju K, Zhuang T, Higashi M, Kolla S, Blobel GA, Brodeur GM The tumour suppressor CHD5 forms a NuRD-type chromatin remodelling complex. Biochem J. 2015:468(2):345-52

35. Kolla V, Zhuang T, Higashi M, Naraparaju K, Brodeur GM. Role of CHD5 in human cancers: 10 years later. Cancer Res. 2014;74(3):652-8.

36. Bagchi A, Papazoglu C, Wu Y, Capurso D, Brodt M, Francis D, Bredel M, Vogel H, Mills AA. CHD5 is a tumor suppressor at human 1p36. Cell. 2007; 128(3):459-75.

\section{Ready to submit your research? Choose BMC and benefit from:}

- fast, convenient online submission

- thorough peer review by experienced researchers in your field

- rapid publication on acceptance

- support for research data, including large and complex data types

- gold Open Access which fosters wider collaboration and increased citations

- maximum visibility for your research: over $100 \mathrm{M}$ website views per year

At BMC, research is always in progress.

Learn more biomedcentral.com/submissions 